\title{
Échec scolaire et didactique de la lecture et de l'écriture
}

Jean-Louis Dumortier

\section{(2) OpenEdition}

1 Journals

Édition électronique

URL : http://journals.openedition.org/ries/4261

DOI : 10.4000/ries.4261

ISSN : 2261-4265

Éditeur

Centre international d'études pédagogiques

Édition imprimée

Date de publication : 1 juin 1994

Pagination : 55-62

ISSN : 1254-4590

\section{Référence électronique}

Jean-Louis Dumortier, «Échec scolaire et didactique de la lecture et de l'écriture », Revue internationale d'éducation de Sèvres [En ligne], 02 | 1994, mis en ligne le 17 avril 2015, consulté le 01 mai 2019. URL: http://journals.openedition.org/ries/4261; DOI : 10.4000/ries.4261

Ce document a été généré automatiquement le 1 mai 2019.

(c) Tous droits réservés 


\title{
Échec scolaire et didactique de la lecture et de l'écriture
}

\author{
Jean-Louis Dumortier
}

\section{Mauvais lecteurs, nos élèves ?}

1 Selon les résultats de la récente enquête sur les compétences en lecture menée dans une trentaine de pays par l'Association internationale pour l'évaluation des rendements scolaires (IEA), les élèves de la Communauté française de Belgique se révèlent, vers l'âge de 9 ans, des lecteurs aux performances très moyennes, et vers l'âge de 14 ans, de très piètres lecteurs puisque la Communauté se classe, pour cette tranche d'âge, en queue du peloton des pays industrialisés.

2 Il n'y a pas de raison de mettre ces résultats sous le boisseau, mais il convient de les interpréter avec plus de circonspection que ceux qui en ont fait état dans la presse. C'est, me semble-t-il, faire preuve d'une excessive désinvolture qu'en déduire l'inefficacité, l'inadaptation radicale de notre pédagogie de la lecture.

En dépit des précautions prises pour que les conditions de passation du test soient rigoureusement identiques dans tous les pays, il se peut qu'elles ne l'aient pas été tout à fait et, en tout état de cause, il n'était pas possible de contrôler qu'elles l'étaient.

4 Par ailleurs, au nombre des faits qui doivent être pris en considération pour expliquer les résultats de nos classes, il y a un triple manque d'accoutumance dont on ne saurait minimiser les effets. Nos élèves ne sont pas habitués à ce genre d'enquête, familier à la population scolaire des pays où se pratique une évaluation centralisée régulière. Pas habitués non plus aux questions à choix multiples qui constituaient l'essentiel du test. Pas habitués, enfin, à traiter des textes informatifs et des documents qui, les uns et les autres, faisaient partie, avec des textes narratifs, de l'échantillon d'écrits sur quoi portaient les questionnaires.

Que l'on me comprenne bien: je ne mets pas en question la qualité du test utilisé, je ne conteste absolument pas qu'il soit un instrument d'évaluation présentant toutes les 
garanties de fiabilité souhaitables. Je dis qu'avec un autre instrument, tout aussi fiable que celui dont on s'est servi, permettant tout aussi bien que lui d'évaluer les performances et d'estimer les compétences, mais portant sur un matériau textuel différent, contenant d'autres formes d'items, on aurait peut-être obtenu des résultats sensiblement différents. Et j'ajoute qu'il faut interpréter ces ceux que fournit l'enquête de l'IEA en tenant compte de l'avantage qu'avaient les élèves des pays où ce genre d'enquête n'a rien d'exceptionnel.

6 À mon avis, on peut donc mettre quelques bémols à la partition catastrophiste que se plaisent à jouer d'aucuns. Mais il n'y a toutefois pas lieu de perpétuer les pratiques pédagogiques traditionnelles dans le détestable confort de la conviction qu'on n'enseigne pas chez nous plus mal qu'ailleurs. Il y a dans notre Communauté, comme chez nos voisins français, comme dans tous les pays industrialisés, quelles que soient les réussites relatives des politiques de «lecturisation " qu'on y mène, une crise de la lecture (ou, moins restrictivement, une crise de la communication écrite) qui constitue un facteur essentiel - peut-être le facteur principal - de l'échec scolaire, étant donné que l'école « fonctionne à l'écrit », contribue à produire et à reproduire une culture de l'écrit.

\section{Échec, écrit et limites d'un consensus}

7 Il serait aisé de faire état d'un très large consensus, établi depuis une vingtaine d'années déjà, sur la nécessité de lutter contre l'échec scolaire, sur l'importance du savoir lire et du savoir-écrire pour la réussite des études, sur l'utilité d'actions visant à donner à la jeunesse les moyens de communiquer par le truchement des écrits ainsi que le goût de le faire. $\mathrm{Du}$ simple citoyen au suprême décideur politique, tous ceux qui ont quelque conscience des besoins économiques du prochain millénaire savent que l'école doit, dès aujourd'hui, prendre en considération la disparition prochaine de la main-d'œuvre non qualifiée, le développement continu du secteur tertiaire et les exigences qu'y manifesteront les employeurs d'un personnel pourvu d'une formation solide (ce qui ne veut nullement dire de capacités spécialisées, bien au contraire!). Tous ceux qui souhaitent, pour les générations à venir, de meilleures conditions de travail et d'existence veulent que l'école soit un instrument de promotion sociale et comprennent, avec plus ou moins de lucidité, qu'elle ne peut l'être sans que les enfants, les adolescents ne développent des compétences solides en matière de lecture et d'écriture. On ne dispose guère d'enquêtes générales sur les représentations de l'écriture et ses pratiques, mais toutes celles qui portent sur la lecture révèlent qu'elle jouit, dans la plus grande partie des couches de la population, d'une image positive et que, dans l'esprit de la majorité des gens, la familiarité avec les livres constitue un gage de réussite scolaire.

Mais un tel consensus ne suffit pas pour élaborer une politique cohérente et efficace visant à développer une culture de l'écrit. Il ne suffit même pas pour mettre au point des actions pédagogiques de longue durée présentant cette cohérence et cette efficacité. Une fois qu'il s'agit de prendre des décisions quant à l'urgence, à l'étendue, à la portée de telle ou telle initiative, le discours consensuel cède vite le pas à des antagonismes de toutes sortes: de la querelle de spécialistes à la querelle de clocher, de la concurrence institutionnelle à la polémique politicienne. En somme, l'unanimité tient à l'usage d'une langue de bois: moins d'échecs et, pour cela, notamment, une meilleure maitrise de l'écrit. Mais sitôt posée la question : que faire ? Les dissensions surgissent, les énergies se dispersent, les entreprises avortent faute de réflexion préalable, de coopération des 
spécialistes, de sensibilisation et de mobilisation des gens de terrain, de suivi et d'évaluation des activités.

9 Pour fonder un tel constat, on peut considérer de multiples aspects de la situation en matière d'éducation à la culture de l'écrit dans la Communauté française de Belgique. Je me contenterai d'évoquer, très rapidement, trois d'entre eux :

- le peu de concertation, de collaboration entre les établissements scolaires et les institutions de lecture publique et, à l'échelon supérieur, entre les ministères dont ils relèvent ;

- l'absence ou la quasi-absence de bibliothèque ou de centre de documentation animé comme il se devrait dans un très grand nombre d'écoles et le peu d'attention consenti, de manière générale, à la question du livre en milieu scolaire (question beaucoup plus vaste que celle de la fourniture de manuels!) ;

- la valse-hésitation des programmes de français qui, jusqu'à il y a très peu de temps, ont toujours souligné le problème de la lecture et cherché des réponses du côté de l'étude des textes (littéraires).

Pour ce qui concerne les deux premiers de ces trois points, il ne me paraît pas douteux que la Communauté française de Belgique puisse prendre exemple sur l'action menée en France, assez récemment, et, depuis plus longtemps, dans divers pays industrialisés. On ne saurait toutefois ignorer que cette action est d'un coût financier certain et que la multiplicité de nos réseaux d'enseignement n'est pas de nature à la faciliter ${ }^{1}$.

\section{Une perspective large sur la crise de la communication écrite}

11 Avant de s'engager dans quelque entreprise que ce soit en vue d'améliorer les compétences des élèves en matière de communication écrite, il faut avoir à l'esprit l'ensemble des facteurs qui déterminent l'état actuel de ces compétences. Il ne s'agit pas, en effet, d'un problème que l'on peut circonscrire en demeurant sur le terrain scolaire et résoudre sur ce terrain-là exclusivement. Qu'on le regrette ou qu'on s'en réjouisse, l'école n'est plus une sorte de monastère, un lieu d'initiation au savoir dont la fréquentation suppose une rupture avec la famille ou le quartier. Quand celui-ci ou celle-là ignorent la communication écrite ou, comme cela se trouve plus souvent, en proclament les mérites sans guère la pratiquer, il ne faut pas s'attendre à ce que, massivement, l'école puisse « convertir» les jeunes à l'écrit. Elle devrait certes proposer des activités susceptibles d'entraîner cette " conversion ", mais non s'illusionner sur son pouvoir de changement. Cela ne signifie pas, bien entendu, que les responsables de l'enseignement puissent se décharger de leurs responsabilités sur les autres responsables de l'éducation; cela signifie que l'efficacité de leurs initiatives est conditionnée par la coopération de toutes les instances concernées par l'action éducative.

La « crise» de la communication écrite telle qu'elle se manifeste aujourd'hui (car l'on aurait beau jeu de montrer qu'il s'agit d'un problème de société récurrent) s'explique tout autant par la croissance des médias audiovisuels et les changements de pratiques de loisirs que par l'inadaptation de l'école à sa nouvelle "clientèle ». Le public d'élèves surgit, à partir des années 70, non seulement de couches sociales «culturellement défavorisées » (c'est-à-dire dont la culture n'est pas celle que diffuse l'école), mais encore de générations affectées par la mise en question des valeurs (morales, esthétiques), par la perte des repères traditionnels, générations qui pouvaient constater que la sélection 
scolaire s'opérait de plus en plus par le truchement des cours scientifiques, celui des mathématiques en particulier.

Concurrencé en tant que moyen d'information et en tant que moyen de distraction, le livre, traditionnellement associé à l'humanisme littéraire, est victime d'un changement de paradigme culturel qui se concrétise, dans l'enseignement secondaire, par la prééminence de fait des filières où dominent les sciences exactes. Toute personne de bonne foi reconnaîtrait que ces dernières (ou tout au moins ce qui s'en enseigne au niveau secondaire) ne sont pas plus «utiles dans la vie» que ne l'étaient les langues anciennes et les lettres classiques ou modernes. Pour l'essentiel, elles jouent le rôle autrefois dévolu à celles-ci comme à celles-là : discriminer, assurer la distinction d'une élite dans une école "démocratisée ». La sélection s'opère désormais sur la base de capacités censées être moins liées à l'habitus culturel des élèves provenant de milieux favorisés, c'est-à-dire moins susceptibles de privilégier ceux que P. Bourdieu et J.C. Passeron avaient désignés, en 1964, comme les « héritiers ». Reste à savoir si, un quart de siècle plus tard, l'héritage n'est pas fort différent de ce qu'il était avant la révolution culturelle qui marque le tournant des années 60-70. Reste à savoir si, mutatis mutandis, l'école ne contribue pas tout autant que par le passé à reproduire certains clivages sociaux - des clivages autrement subtils que l'obsolète dichotomie bourgeoisie/ prolétariat qui a pu servir, il y a trois décennies de cela, d'outil d'analyse, sinon aux « sociologues de la reproduction » qui affirmaient que l'appareil scolaire était conçu pour perpétuer les inégalités sociales, du moins aux vulgarisateurs de leur théorie.

Quoi qu'il en soit, la substitution du couple mathématiques-sciences exactes au couple langues anciennes-français comme instrument de sélection n'a pas changé grand-chose au fait que l'école (et particulièrement l'enseignement secondaire général qui, actuellement, concerne la totalité d'une classe d'âge à un certain moment de son cursus scolaire) diffuse une culture essentiellement livresque dont l'appropriation implique des compétences solides en lecture et (quoique dans une moindre mesure) en écriture. D’où la demande réitérée de tous les enseignants, quelle que soit leur spécialité et à quelque niveau qu'ils enseignent, d'avoir affaire à des apprenants capables de "comprendre ce qu'ils lisent » (autant dire de lire, puisque lire est nécessairement faire sens avec un texte) et de communiquer la pensée qu'ils mettent en forme au moyen du langage écrit.

\section{Situation des enseignants de français}

Cette demande s'adresse, prioritairement, aux professeurs de français dont on ne saurait dire qu'ils disposent aujourd'hui de plus de temps qu'hier pour s'acquitter d'une tâche devenue infiniment plus difficile étant donné la présence massive dans leurs classes d'élèves dont les conduites culturelles extrascolaires font peu de place (ou ne font nulle place) aux pratiques textuelles. On ne saurait faire bon marché de la « crispation » de bon nombre d'enseignants de français qui voient, d'une part, le caractère crucial de leur action reconnu en paroles, et, d'autre part, leur importance négligée concrètement dans les structures pédagogiques et la vie scolaire, étant donné la prééminence de fait des mathématiques et des sciences.

16 L'effet le plus sensible de cette crispation (mais sans doute pas le plus communément reconnu comme tel) est un certain repli sur ce qui, traditionnellement, constitue la spécificité disciplinaire : la grammaire et la littérature. Juste un mot de l'une et de l'autre. 
17 À la faveur d'une rénovation scientifique qui remonte à la fin des années 60 , des pratiques grammaticales purement formelles, inspirées par la linguistique structurale, favorisant la réflexion sur le code mais n'incluant aucune considération sémantique, aucune visée « communicationnelle» ont pu - et peuvent toujours - passer pour une indispensable initiation à la «maîtrise de la langue ». Il serait plus opportun de remplacer cette expression conventionnelle par celle de " compétence discursive " puisque l'on attend des élèves qu'ils puissent utiliser les ressources linguistiques pour émettre et recevoir des textes en situation, autrement dit des discours. Il est plus que douteux que de telles pratiques servent, comme on le prétend, à développer les compétences en lecture et en écriture (encore que certaines connaissances grammaticales soient indispensables à l'exercice de ces compétences), mais beaucoup d'enseignants y sont attachés par réflexe identitaire, notamment.

Quant à la mentalité obsidionale assez répandue dans le milieu des $\mathrm{AESDS}^{2}$ et qui les fait s'ériger en défenseurs du patrimoine littéraire français avec lequel leurs études universitaires les ont tout particulièrement familiarisés, si cette mentalité s'explique par la contestation (aujourd'hui sourde, molle, soft, typiquement postmoderne) des valeurs esthétiques traditionnelles et de l'ascèse requise pour y accéder, elle n'en représente pas moins un obstacle à une réflexion sereine sur la variété des biens culturels disponibles, sur la relativité de toute hiérarchisation de ces derniers et, partant, un obstacle à une action pédagogique qui tiendrait vraiment compte, fût-ce pour les modifier conformément à la mission culturelle de l'école, des représentations et des conduites a priori des élèves.

Pour le dire d'une manière un peu brutale, un peu caricaturale, et sans reconnaître comme elles le mériteraient les multiples initiatives prises par une minorité de professeurs pour affronter les problèmes de lecture et d'écriture qui se posent réellement, il semble bien que de nombreux enseignants de français, dans le souci de préserver l'identité, l'image héritée de leur discipline et de la culture qu'elle représente, s'efforcent, avec souvent beaucoup de conscience professionnelle, de perpétuer des pratiques scolaires dont on a pu autrefois estimer qu'elles conditionnaient le mieux-lire et le mieux-écrire (d'élèves triés sur le volet et déjà pourvus de solides compétences en la matière), mais dont l'efficacité actuelle est pour le moins sujette à caution, sauf à enseigner en quelque milieu privilégié.

\section{Pour une didactique de la lecture et de l'écriture}

Le réflexe de « faire comme on a toujours fait » suppose bien sûr que l'on sache comment l'on a toujours fait. Chaque professeur a ses propres souvenirs d'élève et il ne semble pas qu'au fil des dernières décennies, durant le temps de sa formation initiale, les pratiques qu'il a connues aient fait l'objet d'une mise en question telle qu'il puisse douter de leur efficience une fois qu'il s'est trouvé lui-même en situation d'enseigner.

21 Problème crucial de la formation de base en didactique spéciale, qui ne me semble pas se poser avec une moindre acuité dans les IESP ${ }^{3}$ que dans les universités, et dont on ne saurait affirmer, en l'état actuel des choses, qu'il soit en passe de trouver une solution satisfaisante. Quant à la formation continuée, son efficacité compensatoire ne pourrait s'avérer à grande échelle qu'à la condition d'élaborer des projets d'action d'assez longue 
durée, susceptibles de toucher le plus grand nombre grâce à un système pyramidal de relais, et centrés prioritairement sur des questions de didactique disciplinaire.

À l'heure actuelle, certaines initiatives portent à croire qu'existe quelque méfiance à l'égard des disciplines. Sans aucun doute il convient d'éviter les causes d'échec scolaire liées aux dérives de la spécialisation, de se méfier d'une logique de transfert des connaissances spécialisées conduisant à négliger les représentations des apprenants qui peuvent faciliter ou gêner l'apprentissage de ces connaissances, à ignorer les procédures mentales que l'apprenant utilise. Mais le meilleur moyen de se garder de tels travers, c'est, à mon avis, le développement de la pensée didactique, c'est l'expansion d'une culture didactique permettant à chaque professeur d'envisager les relations entre les trois paramètres de l'action pédagogique: le savoir à enseigner, l'enseignant qui le transpose et l'apprenant qui se l'approprie.

De quelque utilité que soit la réflexion sur les méthodes de travail et autres compétences transversales, il n'en demeure pas moins que tout professeur, étant donné ce que sont actuellement les formations de base, pense sa tâche, conçoit son rôle compte tenu d'un savoir scientifique particulier ou d'un certain nombre de savoirs apparentés. Ce serait déjà, me semble-t-il, un progrès décisif qu'amener l'enseignant à se concevoir non plus en tant que spécialiste d'une discipline, mais en tant que spécialiste de la pédagogie de cette discipline, en tant que didacticien parfaitement conscient du travail qu'il doit accomplir pour donner à l'élève des accès au champ de connaissances dont il est, lui, familier.

S'agissant de lecture et d'écriture (autrement dit des deux composantes les plus importantes du cours de français), le pôle "savoir» du triangle didactique (savoirenseignant-apprenant) est évidemment moins constitué de connaissances déclaratives que de connaissances procédurales: ce sont des savoir-faire que doit développer l'apprenant. Encore cette vision des choses est-elle beaucoup trop limitative : ces savoirfaire sont intimement liés à des attitudes, autrement dit à des dispositions intérieures, qui se manifestent par des réactions émotives face à tel objet ou telle activité. Le succès de l'apprentissage, en ces domaines, n'est pas évaluable uniquement par des performances témoignant des compétences à traiter, selon leurs spécificités, toutes les espèces de textes : il faut en juger également par des indicateurs du fait que les élèves tiennent les écrits pour des objets (différemment pourvus) de valeur, la lecture et l'écriture pour des activités valorisées et valorisantes. Ceci nous ramène à la question des pratiques culturelles et aux problèmes inhérents à un trop grand hiatus entre celles qui ont cours à l'école et celles qui ont cours extra muros.

Quelles que soient les difficultés de leur élaboration, les didactiques de la lecture et de l'écriture constituent, selon moi, à l'heure actuelle, d'indispensables leviers pour s'attaquer au problème de l'échec scolaire. Encore une fois il n'y a pas lieu de croire que leur mise au point suffira à faire de tous les élèves des jeunes qui savent, qui aiment lire et écrire, ni que cette compétence et cette appétence conditionnent à elles seules la réussite. Mais sans elles (que l'on ne gagne rien à mélanger avec d'autres compétences transversales), sans les didactiques qui en assurent le développement, il me paraît vain d'engager la lutte contre l'échec dans une école qui demeure attachée à la culture de l'écrit. 


\section{NOTES}

1. En Communauté française de Belgique coexistent un enseignement libre subventionné $(54,5 \%$ de la population au niveau secondaire) et un enseignement officiel, comprenant lui-même trois réseaux : le premier a pour pouvoir organisateur la commune $(9,7 \%)$, le deuxième, la province $(9,3 \%)$ et le troisième $(26,5 \%)$, l'exécutif de la Communauté elle-même.

2. Agrégés de l'enseignement secondaire du degré supérieur: professeurs formés par les universités qui leur délivrent, outre un titre scientifique, un titre pédagogique d'agrégation, prise en charge par les services de didactique - naguère de méthodologie spéciale) les habilitant à enseigner dans les trois dernières années du secondaire.

3. Instituts d'enseignement supérieur pédagogique. Il s'agit des ex- «écoles normales » qui forment, distinctement, non seulement les maîtres de l'enseignement fondamental (préscolaire et primaire), mais encore les AESDI (agrégés de l'enseignement secondaire du degré inférieur), habilités à donner cours dans les trois premières années du secondaire.

\section{RÉSUMÉS}

Il existe une crise de la communication écrite dont l'école n'est pas la seule responsable. L'auteur évoque l'importance des médias audiovisuels, les changements de pratiques de loisirs, l'école inadaptée à une nouvelle « clientèle » et reproduisant certains clivages sociaux bien plus subtils que jadis. D'où la nécessité de repenser la didactique du français en prenant en compte à la fois le statut général de l'écrit à l'école et les nouvelles données culturelles extra muros.

\section{INDEX}

Index géographique : Belgique

Mots-clés : didactique, échec scolaire, écriture, lecture, didactique du français, langue française

\section{AUTEUR}

\section{JEAN-LOUIS DUMORTIER}

Centre d'auto-formation et de formation continue, Liège. 